\title{
Astrophysical implications of binary black holes in BL Lacertae objects
}

\author{
F. De Paolis, G. Ingrosso, and A. A. Nucita \\ Dipartimento di Fisica, Università di Lecce and INFN Sezione di Lecce, Via Arnesano, CP 193, 73100 Lecce, Italy
}

Received 2 July 2001 / Accepted 25 March 2002

\begin{abstract}
Some BL Lacertae objects show a periodic behaviour in their light curves that is often attributed to the orbital motion of a central binary black hole system. On this basis, and assuming a circular orbit, Rieger \& Mannheim (2000) have recently proposed a method to determine the orbital parameters of the binary system from the observed quantities, i.e. the signal periodicity, the flux ratio between maximum and minimum signal and the power law spectral index of the photon flux. However, since these binary black holes are expected to originate from galactic mergers, they could well be on eccentric orbits, which might not circularize for a substantial time. We therefore generalize the treatment proposed by Rieger \& Mannheim (2000) by taking into account the effect of the orbital eccentricity of the binary system. We apply the model to three well-observed Markarian objects: MKN 501, MKN 421 and MKN 766 that most likely host a binary system in their centers. Some astrophysical implications of this model are also investigated with particular emphasis to the gravitational radiation emission from the binary black holes. Under particular conditions (e.g. for some values of the orbital separation, eccentricity and Lorentz factor) one can obtain signals above the sensitivity threshold of the LISA detector.
\end{abstract}

Key words. BL Lacertae objects: general - black hole physics

\section{Introduction}

BL Lacertae objects, also known as Markarian objects (hereafter MKNs), belong to the class of active galaxies. According to the well-established unified model on radioloud active galactic nuclei (see Urry \& Padovani 1995), these objects are thought to be dominated by relativistic jets seen at small angles to the line of sight. The structure of the relativistic jets is largely unknown and the typical smooth and fairly featureless spectra of such objects may be reproduced by models making use of very different assumptions. Combining spectral and temporal informations greatly constrains the available models.

Up to now, several astrophysical phenomena have been attributed to binary black holes, like precession of jets (Begelman et al. 1980), misalignment (Conway \& Wrobel 1995), periodic outburst activity in the quasar OJ 288 (Sillanpää et al. 1988; Letho \& Valtonen 1996) and precession of the accretion disk under gravitational torque (Katz 1997).

It has been recently observed that some MKN objects show a periodic behaviour in the radio, optical, X-ray and $\gamma$-ray light curves that is possibly related to the presence

Send offprint requests to: F. De Paolis,

e-mail: Francesco.DePaolis@le.infin.it of a massive binary black hole creating a jet either aligned along the line of sight or interacting with an accretion disk (Yu 2001). Therefore, the search for X-ray $/ \gamma$-ray variability can be considered as a method to probe the existence of a massive binary black hole in the center of a galaxy.

Massive black hole systems are expected to be fairly common in the Universe as a result of merging between galaxies. Indeed, in the framework of the unified model for the morphological evolution of galaxies (see e.g. White 1997) giant ellipticals, which are the host galaxies of most MKN objects, appear to be the result of mergers between spirals. On the other hand, it is now well established that galaxies generally contain massive black holes in their nuclei (Rees 1984; Kormendy \& Richstone 1995; Richstone et al. 1998). Therefore, merging would naturally lead to the formation of massive binary black holes (Begelman et al. 1980).

At least three MKN objects (i.e. MKN 501, MKN 421 and MKN 766) are particularly well studied at high energies, revealing a possible periodic behaviour in their light curves.

MKN 501, at $z=0.034$, shows a clear well-correlated 23 day periodicity in $X$-ray and $\mathrm{TeV}$ energy bands with an observed TeV flux ratio $f \simeq 8$ between the maximum and 
minimum of the signal (Protheroe et al. 1998; Hayashida et al. 1998; Kranich et al. 1999; Nishikawa et al. 1999), while evidence for correlations in the optical $U$-band is rather weak (Catanese et al. 1997; Aharonian et al. 1999). It has also been suggested that the complex morphology of the jet and the peculiar behaviour of its spectral energy distribution are probably related to the presence of a massive binary black hole (Conway \& Wrobel 1995; Villata \& Raiteri 1999).

MKN 421, at $z=0.031$, is the brightest BL Lacertae object at X-ray and UV wavelengths and it is the first extragalactic source discovered at $\mathrm{TeV}$ energies (Punch et al. 1992). This nearby source, which has been recently observed by the XMM-Newton (see Brinkmann et al. 2000) and by BeppoSax (Maraschi et al. 1999) satellites, shows remarkable $X$-ray variability correlated with strong activity at $\mathrm{TeV}$ energies (George et al. 1988) on a time-scale of $\simeq 10^{4} \mathrm{~s}$ (Maraschi et al. 1999) and with a flux ratio $f \simeq 2$.

X-ray observations of the nearby MKN 766, at $z=$ 0.013 , have been performed by the XMM-Newton satellite (Boller et al. 2000). These observations have revealed the presence of a strong X-ray periodic signal with frequency $\simeq 2.4 \times 10^{-4} \mathrm{~Hz}$ and flux ratio $f \simeq 1.3$.

Based on the assumption that the periodic observed light curve of MKN 501 is related to the presence of a binary system of black holes (one of which emits a jet), Rieger \& Mannheim (2000) have proposed a method to determine the physical parameters of the binary system from the observed quantities, i.e. signal periodicity, flux ratio between maximum and minimum signal and power law spectral index. They adopted the simplifying assumptions that $i$ ) the two massive black holes move on circular orbits around the common center of mass and ii) the binary separation $a$ is such that the gas dynamical time-scale $T_{\text {gas }}$ is equal to the time-scale for gravitational radiation $T_{\mathrm{gw}}$ (see also Begelman et al. 1980).

However, if binary black holes originated from galactic mergers, they could be on eccentric orbits and eccentricity values up to $0.8-0.9$ are not necessarily too extreme (Fitchett 1987). Of course, due to gravitational wave emission, orbits tend to circularize but this happens within a time-scale of the same order of magnitude as the merging time-scale (Peters 1964; Fitchett 1987). Therefore, if a massive binary black hole is found at the center of a galaxy, it may happen that the constituting black holes are still on eccentric orbits, in which case the method proposed by Rieger \& Mannheim (2000) does not hold.

As far as point ii) is concerned, we note that the assumption that nowadays $T_{\text {gas }}$ is equal to $T_{\mathrm{gw}}$ is arbitrary. Indeed, it is possible that $T_{\mathrm{gas}}=T_{\mathrm{gw}}$ at some time in the past, but since then the binary system evolution was driven by gravitational wave emission which shrunk the orbital separation faster (see Sect. 2).

The aim of the present paper is to generalize the model proposed by Rieger \& Mannheim (2000) by taking into account the effects of the orbital eccentricity of the binary system and considering the binary separation $a$ as a free model parameter. This is done in Sect. 2, where the model is also applied to three well-observed MKN objects (MKN 501, MKN 421 and MKN 766) obtaining a set of possible values for the free model parameters $\gamma_{\mathrm{b}}, a$ and $e$ corresponding to different values for the masses $m$ and $M$ of the two black holes. In Sect. 3 we study the gravitational waves emitted by the binary systems at the center of the above-quoted MKN objects and analyze the possibility of detecting them by the LISA gravitational wave interferometer. Our conclusions are presented in Sect. 4.

\section{Binary black hole model for BL Lacertae objects}

In the commonly-accepted evolutionary scenario, giant elliptical galaxies are believed to be the product of mergers between spiral galaxies. Since each galaxy likely contains a central black hole with mass $10^{6}-10^{9} M_{\odot}$ (Richstone et al. 1998), merging would sometimes produce binary black hole systems separated by a typical distance of $0.1-1 \mathrm{pc}$ (Begelman et al. 1980).

We assume that binary black holes exist in the center of many MKN objects and in particular in those of MKN 501, MKN 421 and MKN 766. As in Rieger \& Mannheim (2000), the periodicity in the flaring state, observed towards these objects, is assumed to be the consequence of the orbital motion of a relativistic jet in the binary black hole. Therefore, the observed signal periodicity has a geometrical origin, being a consequence of Dopplershifted modulation. Let us further assume that the jet is emitted by the less massive black hole and that the nonthermal X-ray $/ \gamma$-ray radiation propagates outwards from the core along the jet with Lorentz gamma factor $\gamma_{\mathrm{b}}$. The observed flux modulation due to Doppler boosting can be written as

$S(\nu)=\delta^{3+\alpha} S^{\prime}(\nu)$

where $\alpha$ is the source spectral index ${ }^{1}$ and the Doppler factor is given by

$\delta=\frac{\sqrt{1-\left(v_{z}^{2}+v_{\mathrm{ls}}^{2}\right) / c^{2}}}{1-\left(v_{z} \cos i+v_{\mathrm{ls}} \sin i\right) / c}$

Here, $v_{z}$ is the outflow velocity in the direction of the total angular momentum, $i$ is the inclination angle between the jet axis and the line of sight and $v_{\mathrm{ls}}$ is the component of the less massive black hole velocity along the line of sight.

Let $M$ and $m$ represent the masses of the primary and the secondary black hole, respectively. Conventionally, the problem of predicting the motion of a system of two gravitationally interacting point masses is simplified by considering the equivalent system. This consists of a mass $M_{\mathrm{t}}=M+m$ fixed in space and acting on a reduced

${ }^{1}$ Values of the power law index $\alpha$ for the three MKNs of interest are found to be 1.2, 1.7 and 2.11 for MKN 501, MKN 421 and MKN 766, respectively. For more details see Rieger \& Mannheim (2000), Guainazzi et al. (1999) and Boller et al. (2000). 
mass $\mu=M m /(M+m)$ that orbits around it. We assume that the reduced mass moves around the total mass on an elliptic orbit with semi-major axis $a$ and eccentricity $e$. Let $(r, \theta)$ be the polar coordinates of $\mu$ relative to $M_{\mathrm{t}}$, so that the equation of the orbit is given by

$r=\frac{a\left(1-e^{2}\right)}{1+e \cos \theta}$.

It is straightforward to demonstrate that the component of the reduced mass velocity along the line of sight is given by (Smart 1977)

$v_{\mu, \mathrm{ls}}=\sqrt{\frac{G(M+m)}{a\left(1-e^{2}\right)}} \sin \theta$.

The component of the less massive black hole velocity along the line of sight is thus given by

$v_{\mathrm{ls}}=\frac{M}{M+m} v_{\mu, \mathrm{ls}}$,

which can be rewritten as

$v_{\mathrm{ls}}=R_{\mathrm{a}} \Omega_{\mathrm{a}} \frac{\sin \theta}{\left(1-e^{2}\right)^{\frac{1}{2}}}$,

where $R_{\mathrm{a}}=M a /(M+m)$ and $\Omega_{\mathrm{a}}=\left[G(M+m) / a^{3}\right]^{1 / 2}$ is the Keplerian orbital frequency if the mass $m$ moves on a circular orbit of radius $a$ around $M$. The velocity $v_{\mathrm{ls}}$ is maximal for $\theta=\pi / 2$ and minimal for $\theta=3 \pi / 2$, corresponding through Eq. (2) to the two possible values $\delta_{\max }$ and $\delta_{\min }$ of the Doppler factor.

With the assumption that the periodicity in the observed signal is due to the orbital motion of the binary black hole, from Eq. (1) one obtains the condition $\delta_{\max } / \delta_{\min } \simeq f^{1 /(3+\alpha)}$, where $f$ is the observed maximum to minimum flux ratio. Consequently, by using Eqs. (2) and (6) we have

$\Omega_{\mathrm{a}} R_{\mathrm{a}}=\frac{f^{1 / 3+\alpha}-1}{f^{1 / 3+\alpha}+1}\left(\frac{1}{\sin i}-\frac{v_{z}}{c} \cot i\right)\left(1-e^{2}\right)^{\frac{1}{2}} c$,

which, in the limit $e \rightarrow 0$, reduces to Eq. (4) in Rieger \& Mannheim (2000).

As a consequence of the binary orbital revolution around the center of mass, the observed signal period $P_{\text {obs }}$ is related to the keplerian period $P_{\mathrm{k}}=2 \pi / \Omega_{\mathrm{a}}$ by (Rieger \& Mannheim 2000)

$P_{\mathrm{obs}}=(1+z)\left(1-\frac{v_{z}}{c} \cos i\right) P_{\mathrm{k}}$,

where $i \simeq 1 / \gamma_{\mathrm{b}}$ and $v_{z} \simeq c\left(1-1 / \gamma_{\mathrm{b}}^{2}\right)^{1 / 2}($ Spada 1999). From Eqs. (7) and (8), one obtains the following relation

$$
\begin{aligned}
& \frac{M}{(m+M)^{2 / 3}}=\frac{P_{\mathrm{obs}}^{1 / 3}}{[2 \pi(1+z) G]^{1 / 3}} \frac{c}{\sin i} \\
& \quad \times \frac{f^{1 / 3+\alpha}-1}{f^{1 / 3+\alpha}+1}\left(1-\frac{v_{z}}{c} \cos i\right)^{2 / 3}\left(1-e^{2}\right)^{1 / 2},
\end{aligned}
$$

which, in the limit $e \rightarrow 0$, reduces to Eq. (8) of Rieger \& Mannheim (2000).
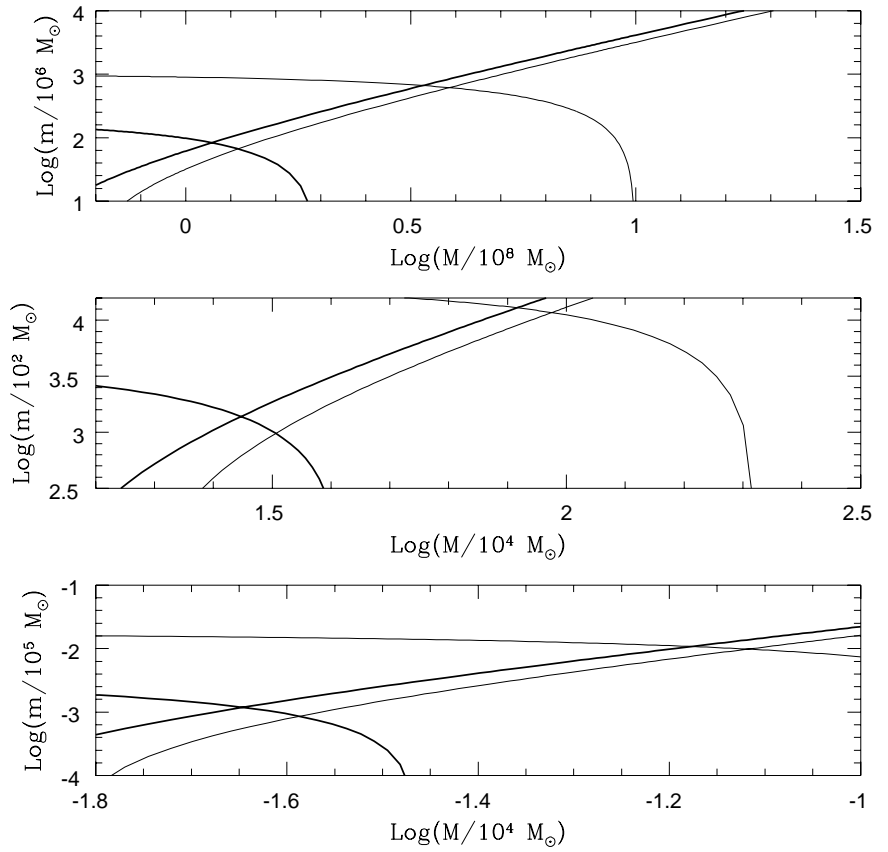

Fig. 1. From the upper to the bottom panel, the required mass dependence for binary black hole model is shown in the case of MKN 501, MKN 421 and MKN 766, respectively. The thick and thin lines represent the conditions expressed in Eqs. (9) and (10) with Lorentz factor $\gamma_{\mathrm{b}}=10$ and $\gamma_{\mathrm{b}}=15$. The binary separation $a$ is set to $5 \times 10^{16} \mathrm{~cm}, 2 \times 10^{14} \mathrm{~cm}$ and $1 \times 10^{13} \mathrm{~cm}$ for MKN 501, MKN 421 and MKN 766, respectively. We have set the orbit eccentricity value equal to 0.5 . The intersection between lines corresponding to the same Lorentz factor gives the masses of the black holes in the binary system considered.

Moreover, from Eq. (8) one has

$m+M=\left[\frac{2 \pi(1+z)\left(1-\frac{v_{z}}{c} \cos i\right)}{P_{\mathrm{obs}}}\right]^{2} \frac{a^{3}}{G}$

where $a$ represents the semi-major axis of the binary system.

Therefore, the allowed primary and secondary black hole masses $m$ and $M$ are obtained by solving simultaneously Eqs. (9) and (10). In these equations $\gamma_{\mathrm{b}}, e$ and $a$ have to be considered as free model parameters to be determined by the observed data $\left(P_{\text {obs }}, f\right.$ and $\left.\alpha\right)$ towards the three MKK of interest. In this way, we can estimate the masses of the black holes supposed to be at the centers of MKN 501, MKN 421 and MKN 766.

We mention that Eq. (10) is different from the corresponding one in Rieger \& Mannheim (2000), where the binary separation $a$ is obtained by equating the gas dynamical time-scale (Begelman et al. 1980) with the timescale for gravitational radiation (Peters \& Mathews 1963). Here, as previously stated, we release this assumption and consider $a$ as a free fit parameter.

For the three MKN objects considered, the secondary mass $m$ as a function of the primary one $M$ is shown in Fig. 1 for selected values of $\gamma_{\mathrm{b}}=10,15$ and $e=0.5$. For each binary system, the intersection between lines with 


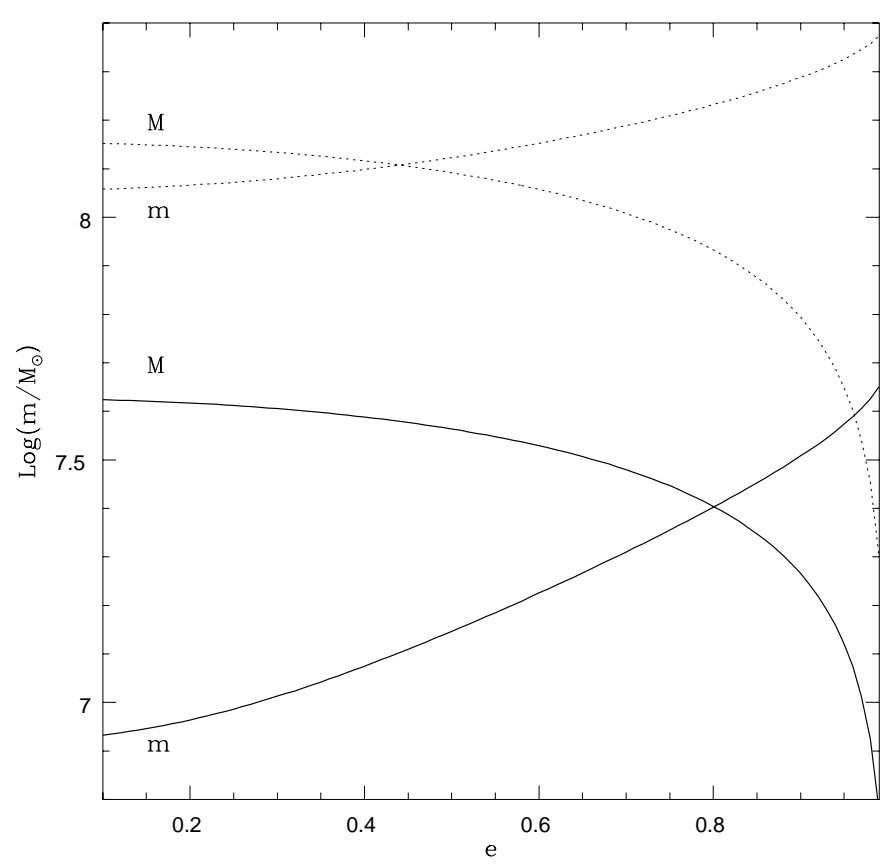

Fig. 2. The dependence of the primary and secondary mass on the eccentricity of the orbit is shown for MKN 501 Lorentz $\gamma_{\mathrm{b}}$ factors $\gamma_{\mathrm{b}}=10$ (dotted lines) and $\gamma_{\mathrm{b}}=30$ (solid lines), respectively. Here we have set $a=8 \times 10^{16} \mathrm{~cm}$. Similar plots can be obtained for the MKN 421 and MKN 766 .

the same Lorentz factor determines the masses of the two black hole components.

In Fig. 2 (for MKN 501) we show the primary and secondary black hole masses as a function of the orbit eccentricity for two values of the Lorentz factor $\gamma_{\mathrm{b}}=10,30$ and $a=8 \times 10^{16} \mathrm{~cm}$. In this case, the black hole masses are in the range $10^{6}-10^{9} M_{\odot}$.

\section{Gravitational waves from massive black hole binaries in Markarian objects and comparison with LISA sensitivity}

In this section, the massive black hole binaries in the MKN objects considered in Sect. 2 are examined as possible sources of gravitational radiation. Due to the orbital period of the binary systems considered, it is expected that they emit most of the gravitational radiation in the low-frequency band $\left(\nu \lesssim 10^{-4} \mathrm{~Hz}\right)$. Gravitational waves in this band are only accessible to experiments based on the Doppler tracking technique of inter-planetary spacecrafts. The Laser Interferometer Space Antenna (LISA), consisting of a constellation of three drag-free spacecrafts at the vertices of an ideal equilater triangle with sides of $\sim 5 \times 10^{6} \mathrm{~km}$ (Hiscock et al. 2000), has an optimal sensitivity in the frequency range covering the band $10^{-5} \mathrm{~Hz}^{-}$ $3 \times 10^{-2} \mathrm{~Hz}$ (Vecchio 1999). LISA will be able to carry out a deep and extensive census of black hole populations in the Universe, providing an accurate demography of these objects and their interactions. In particular we expect that binaries of massive black holes could be detectable by LISA even if the emitted gravitational waves are charac- terized by extremely low frequencies $\left(\nu \lesssim 10^{-4} \mathrm{~Hz}\right)$. This could be the case for the binaries at the center of the three MKN objects considered under particular conditions (e.g. for the same values of $a, e$ and $\gamma_{\mathrm{b}}$ ).

Indeed we expect that in a merging process between galaxies, the two colliding black holes at their centers would eventually move on orbits with non-negligible eccentricity. This fact should have great importance as far as the detection of the emitted gravitational waves is concerned. In fact, a binary system with an eccentric orbit emits significantly more power in gravitational waves than a circular system with the same semimajor axis. Moreover, eccentric binaries will also emit gravitational waves in a multitude of harmonics of the orbital frequency $\omega_{\mathrm{k}}$ associated with the semimajor axis $a$. Thus, an eccentric system emits gravitational waves at the frequencies $\omega_{n}=n \omega_{\mathrm{k}}$ (with $n=1,2,3 \ldots$ ), whereas circular binaries emit purely in the $n=2$ mode. Peters \& Mathews (1963), found that the orbit averaged power emitted in gravitational waves by a binary system with eccentricity $e$ in the $n$th harmonic of the orbital frequency is

$$
\frac{\mathrm{d} E(n, e)}{\mathrm{d} t}=\frac{32}{5} \frac{G^{4} M^{2} m^{2}(M+m)}{c^{5} a^{5}} g(n, e),
$$

where the function $g(n, e)$ depends on $n$ and $e$ by the following relation

$$
\begin{aligned}
g(n, e)= & \frac{n^{4}}{32}\left\{\left[J_{n-2}(n e)-2 e J_{n-1}(n e)\right.\right. \\
& \left.+\frac{2}{n} J_{n}(n e)+2 e J_{n+1}(n e)-J_{n+2}(n e)\right]^{2} \\
& +\left(1-e^{2}\right)\left[J_{n-2}(n e)-2 J_{n}(n e)+J_{n+2}(n e)\right]^{2} \\
& \left.+\frac{4}{3 n^{2}}\left[J_{n}(n e)\right]^{2}\right\}
\end{aligned}
$$

Here $J_{n}$ (with $n$ integer) is the usual Bessel function of order $n$ th. The characteristic spectral amplitude at the frequency $\omega_{n}$ can be evaluated as

$h\left(\omega_{n}\right) \simeq \frac{2}{\pi \omega_{n} D} \sqrt{\frac{G}{c^{3}} \frac{\mathrm{d} E(n, e)}{\mathrm{d} t}}$,

where $D$ is the distance of the gravitational wave source from Earth.

Equations (11)-(13) allow us to evaluate the expected spectrum of the gravitational waves emitted by the massive black hole binaries assumed to be in the center of the considered MKN objects. Assuming $\gamma_{\mathrm{b}}=5$, Fig. 3 shows the expected gravitational wave amplitude $h$ as a function of the frequency $\omega$, for different values of the binary orbit eccentricity. In each panel of the figure we give for comparison the LISA sensitivity curve (the oblique solid line) for an integration time of 5 years. As can be noted, in several cases the expected gravitational waves emitted by the considered binary systems may be detected by LISA satellite. Eccentricity values up to 0.8 are not necessarily too 

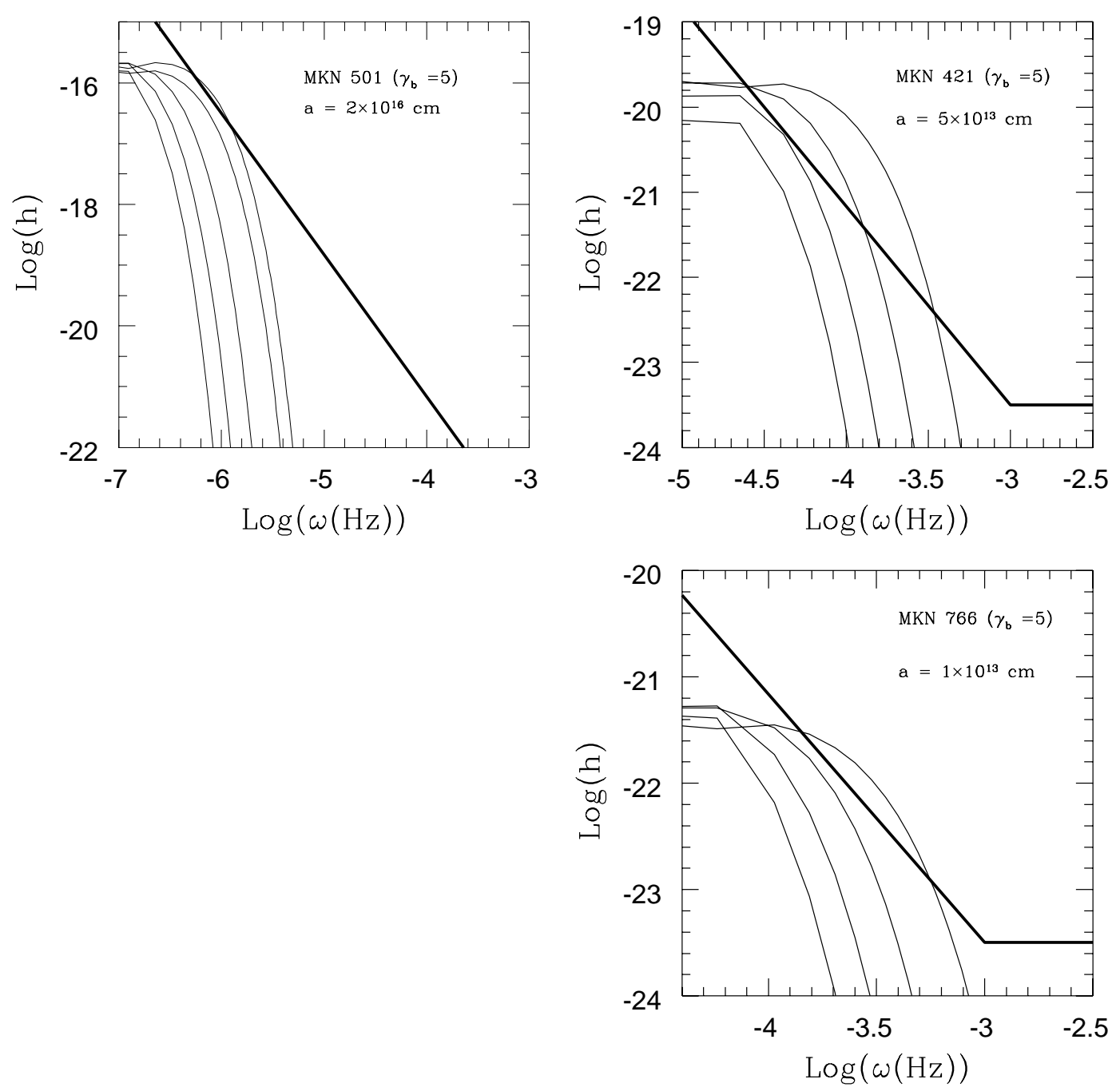

Fig. 3. The amplitude of the gravitational waves emitted by the binary black holes fitting all the observational data is reported as a function of the frequency for the three MKN objects considered. The continuous lines from left to right in each panel correspond to orbital eccentricity $e=0.5,0.6,0.7,0.8$, respectively (for MKN 501 we added a curve corresponding to $e=0.85$ ). The chosen orbital radius $a$ is indicated in each panel. The solid oblique line represents the LISA sensitivity with an integration time of 5 years.

extreme. In fact, if a black hole binary forms in a merging process, a very high value of eccentricity is expected. Of course, due to the gravitational wave emission the orbit tends to circularize but this should happen within a timescale of the same order of the magnitude of the merging time-scale (Peters 1964).

In Fig. 4, the binary semimajor axis $a$ is shown as a function of the orbital eccentricity $e$ and for two different Lorentz factors $\gamma_{\mathrm{b}}$ for MKN 501, MKN 421 and MKN 766, respectively. Each point in the 3D scatter plot fits the observations and satisfies Eqs. (9) and (10). Each point on the plot corresponds to a different distance $d=h\left(\omega_{n}\right) / h_{\text {LISA }}\left(\omega_{n}\right)$ (vertical axis) between the gravitational wave emitted spectrum and the LISA sensitivity curve for an integration time of 5 years (the minimum distance between the curves diminishes as $d$ increases). In Fig. 4, only models with $\inf d\left(\omega_{n}\right) \geq 0.1$ are shown. Models corresponding to $d \geq 1$ in the plot have to be considered as observable by the LISA satellite. We notice that models corresponding to higher values of the parameter $d$ have typically higher masses and lower coalescing times with respect to those with lower $d$ values. In any case, black hole masses are below $10^{9} M_{\odot}$.

\section{Discussion}

We have considered some astrophysical implications of the existence of massive black hole binary systems at the center of MKN objects. These systems are expected to be fairly common in the Universe as a consequence of merging processes between galaxies. In fact, following the evolutionary scenario, giant elliptical galaxies are thought to be the result of merging between spirals. Since almost each galaxy contains a massive black hole in its center, merging process would naturally lead to the formation of massive black hole binaries.

Currently, the only method to probe the existence of massive binary black holes is to search for double nuclei 

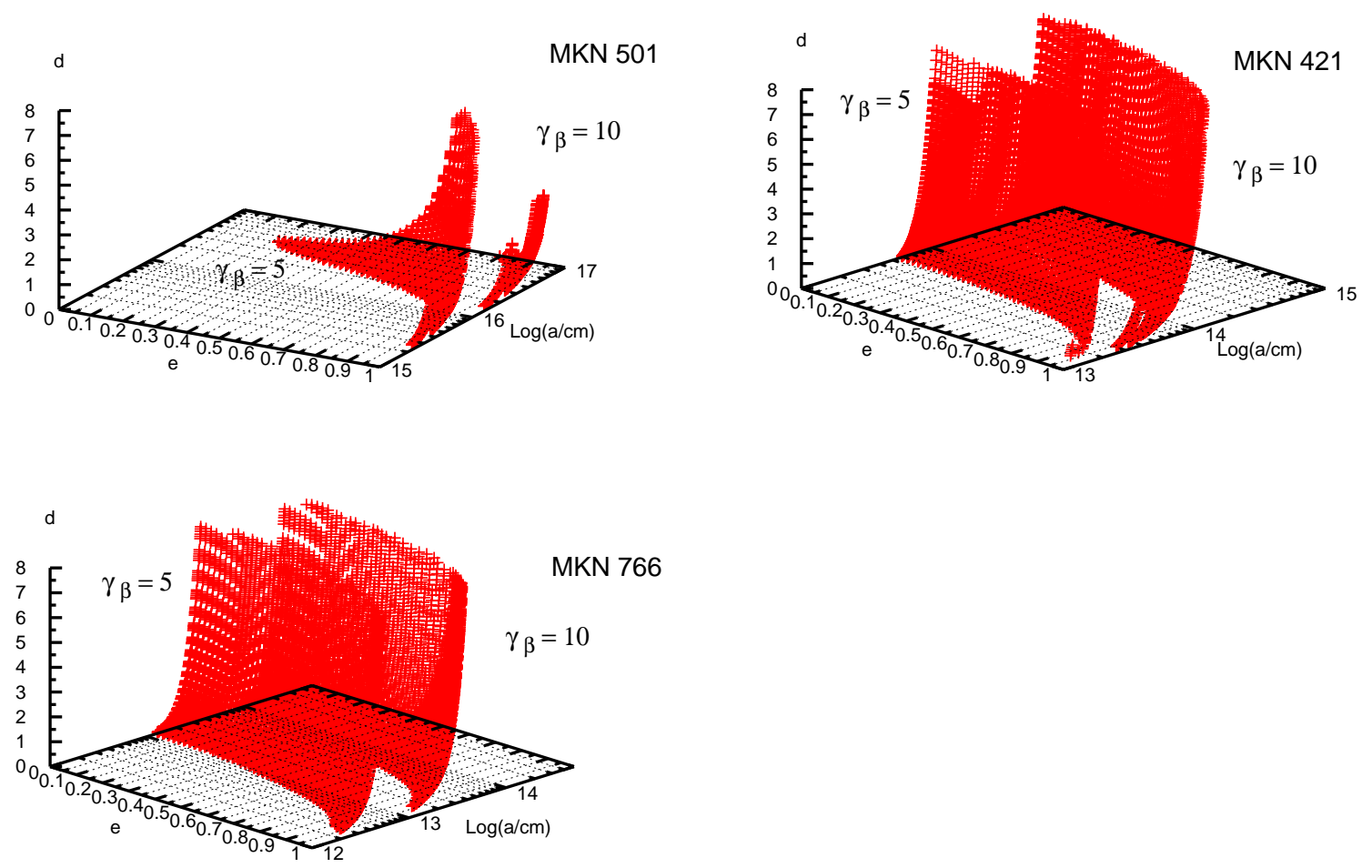

Fig. 4. In the 3D scatter plot, the binary semimajor axis $a$ is shown as a function of the orbital eccentricity $e$ for different values of Lorentz factor $\gamma_{\mathrm{b}}$, for MKN 501, MKN 421 and MKN 766, respectively. Each point in the evidenced regions of the $a-e$ plane fits the observations and satisfies Eqs. (9) and (10). On the vertical axis, the detection parameter $d$ is also shown (for details see text).

in the galactic centers. However, as pointed out by Rieger \& Mannheim (2000) and $\mathrm{Yu}$ (2001), a periodic behaviour in the observed radio, optical, X-ray or $\gamma$-ray light curves could be considered as the signature of a massive binary black hole.

In this paper, we focused on the possibility of explaining the intensity variations observed in some MKN objects as the consequence of the motion of binary black holes around the common center of mass. We have generalized the treatment of Rieger \& Mannheim (2000) considering in detail the effects of the orbital eccentricity.

At least three MKN objects (MKN 501, MKN 421 and MKN 766) reveal a possible periodic behaviour in the observed signal. In particular, it has been recently addressed whether MKN 501 hosts a massive black hole binary system (Rieger \& Mannheim 2000). The same likely holds for MKN 421 and MKN 766. The model described in Sect. 2 allows us to estimate the mass of each black hole in the binary system (see Fig. 1) as a function of the Lorentz factor $\gamma_{\mathrm{b}}$, of the orbital radius $a$ and eccentricity $e$.

If massive binary black holes really exist in the centers of the MKN objects considered above, they should also emit low frequency gravitational waves typically with $\omega \lesssim 10^{-4} \mathrm{~Hz}$. In Fig. 3 we have presented the gravitational wave spectrum emitted by binaries that fit all the observational data (flux ratio $f$, the signal periodicity $P_{\text {obs }}$ and the source spectral index $\alpha$ ). As one can note, for some values of $a$ and $e$ one can obtain curves above the sensitivity threshold of the LISA detector (we assume an integration time of 5 years).

It is obvious that binary systems emitting gravitational waves detectable by the LISA satellite have short coalescing times. For example, in the case of MKN 501 the average coalescing time for the models represented in Fig. 4 (for $\gamma_{\mathrm{b}}=5$ ) is $\sim 10^{3}$ years and tends to decrease for higher values of the parameter $d$.

However, for signal detection, some caution is needed since the LISA sensitivity curve we use in Figs. 3 and 4 does not take into account the noise from the galactic binaries containing two compact objects. Especially important are galactic white dwarf binaries (Hils \& Bender 2000; Nelemans et al. 2001; Nelemans et al. 1999); primordial black holes (Hiscock 1998) and white dwarf binaries (Hiscock et al. 2000) in the galactic halo. These binaries would give rise to a confusion noise which lies well above LISA's sensitivity curve in the frequency range of interest between $10^{-4} \mathrm{~Hz}$ and $10^{-3} \mathrm{~Hz}$ (see e.g. Fig. 3 in Nelemans et al. 2001). This noise would make even more difficult the detection of the considered massive black hole binary systems so that, in order to have detectable signals, one would need more extreme conditions (e.g. higher values of the parameter $d$ or, equivalently, shorter coalescing times).

As a final remark we notice that it is possible that there are a lot of hard binary black holes at the center of galaxies, MKN objects, Seyfert galaxies, etc., and that we can observe directly (from the periodicity of lightcurves at high energy) only a small fraction of them. 
If this is so, detection of gravitational waves from such systems would be a means to detect them. It is worth noting that the number of intervening galaxies between Earth and a galaxy at redshift $z$ is

$N \simeq 6 \times 10^{-3}\left(\frac{R}{3 \mathrm{kpc}}\right)^{2}\left(\frac{N_{\mathrm{g}}}{0.1 \mathrm{Mpc}^{-3}}\right)\left[(1+z)^{3 / 2}-1\right]$,

where $R$ is the typical galaxy radius and $N_{\mathrm{g}}$ the average number density of galaxies. Therefore, it is expected that at least in some cases gravitational lenses, displaced along the line of sight of a gravitational wave source, should distort the path of the gravitational waves just as they distort the path of the observed light. For gravitational waves with frequency $\nu \lesssim 10^{-4} \mathrm{~Hz}$, the LISA angular resolution is below $10^{-2}$ steradians (Cutler 1998). Consequently, the lensed source is always seen as an amplitude amplification (i.e. as a microlensing event) and the observed amplitude, at the frequency $\omega_{n}$, is (De Paolis et al. 2001)

$h^{\max }\left(\omega_{n}\right) \simeq \sqrt{A^{\max }} h\left(\omega_{n}\right)$

where $A^{\max }$ is the amplification parameter depending on the geometry of the microlensing configuration. This amplification could be in some cases of the order of 10 or more, thereby increasing the number of detectable binary black holes at galactic centers by future gravitational wave detectors like LISA. Even more interesting would be the effect of the gravitational wave time-delay due to the different paths traveled by the deflected signals. This timedelay, of about one year as for the lensed Quasars, would give rise to a frequency difference due to the binary evolution with time. This effect could produce a "beating pattern" in the wave amplitude which could be searched for in future gravitational wave experiments.

Acknowledgements. We would like to thank Prof. Asghar Qadir for interesting discussions about the problem. We also warmly acknowledge an anonymous referee for pointing out some important issues.

\section{References}

Aharonian, F., Akhperjanian, A. G., Barrio, J. A., et al. 1999, A\&A, 349, 29

Begelman, M. C., Blandford, R. D., \& Rees, M. J. 1980, Nature, 287,307

Boller, T., Keil, R., Trümper, J., et al. 2001, A\&A, 365, 134

Brinkmann, W., Sembay, S., Griffiths, R. G., et al. 2001, A\&A, 365,162

Catanese, M., Bradbury, S. M., Beslin, A. C., et al. 1997, ApJ, 487, L143

Conway, J. E., \& Wroble, J. M. 1995, ApJ, 439, 98

Cutler, C. 1998, Phys. Rev. D, 57, 7089

De Paolis, F., Ingrosso, G., \& Nucita, A. A. 2001, A\&A, 366, 1065
Fitchett, M. 1987, MNRAS, 224, 567

George, I. M., Warwick, R. S., \& Bromage, G. E. 1988, MNRAS, 232, 793

Guainazzi, M., Vacanti, G., Malizia, A., et al. 1999, A\&A, 342, 124

Haehnelt, M. G. 1998, Laser Interferometer Space Antenna, Second Int. LISA Symp. on the Detection and Observation of Gravitational Waves in Space, ed. W. M. Folkner, AIP Conf. Proc., 456, 45

Hayashida, N., Hirasawa, H., Ishikawa, F., et al. 1998, ApJ, 504, L71

Hiscock, W. A. 1998, ApJ, 509, L101

Hiscock, W. A., Larson, S. L., Routzahn, J. R., \& Kulick, B. 2000, ApJ, 540, L5

Katz, J. I. 1997, ApJ, 478, 527

Kormendy, J., \& Richstone, D. 1995, ARA\&A, 33, 581

Kranich, D., deJager, O. C., Kestel, M., et al. 1999, in Proc. of 26th International Cosmic Ray Conference (Salt Lake City), 3, 358

Hils, D., \& Bender, P. L. 2000, ApJ, 537, 334

Letho, H. L., \& Valtonen, M. J. 1996, ApJ, 460, 207

Maraschi, L., Fossati, G., Tavecchio, F., et al. 1999, Astropart. Phys., 11, 189

Molendi, S., Maccaro, T., \& Schaeidt, S. 1993, A\&A, 271, 18

Nelemans, G., Portegies-Zwart, S. F., \& Verbunt, F. 1999, preprint [astro-ph/9903255]

Nelemans, G., Yungelson, L. R., \& Portegies-Zwart, S. F. 2001, A\&A, 375, 890

Nishikawa, D., Hayashi, S., Chamoto, N., et al. 1999, in Proc. of 26th International Cosmic Ray Conference (Salt Lake City), 3, 354

Peters, P. C. 1964, Phys. Rev., 136, 1224

Peters, P. C., \& Mathews, J. 1963, Phys. Rev., 131, 435

Protheroe, R. J., Bhat, C. L., Fleury, P., et al. 1998, in Proc. 25th International Cosmic Ray Conference (Durban) 8, 317

Punch, M., Akerlof, C. W., Cawley, M. F., et al. 1998, Nature, 358,477

Rees, M. 1984, ARA\&A, 22, 471

Richstone, D., Ajhar, E. A., \& Bender, R. 1998, Nature, 395, 14

Rieger, F. M., \& Mannheim, K. 2000, A\&A, 359, 948

Shapiro, S. L., \& Teukolsky, S. A. 1983, Black Holes, White Dwarfs, and Neutron Stars (John Wiley and \& Sons, New York)

Smart, W. M. 1977, Textbook on Spherical Astronomy (Cambridge University Press, Cambridge)

Spada, M. 1999, Astropart. Phys., 11, 59

Sillanpää, A., Haarala, S., Valtonen, M. J., et al. 1988, ApJ, 325,628

Urry, L. M., \& Padovani, P. 1995, PASP, 107, 803

Vecchio, A. 1999, in Gravitational Waves, Third E. Amaldi Conference, AIP Conf. Proc. 523 (AIP Press), 238

Villata, M., \& Raiteri, C. M. 1999, ApJ, 347, 30

White, S. D. M., in ed. G. Börner, \& S. Gottlober, The evolution of the Universe: Report of the Dahlem Workshop (Berlin, 1997), 227

Yu, Q. 2001, preprint [astro-ph/0109530]

Yu, Q., \& Lu, Y. 2001, A\&A, 377, 17 